\title{
PATHOLOGICAL FEATURES AND GENETIC TESTING AMONG BREAST CANCER YOUNG WOMEN: A SINGLE-INSTITUTION EXPERIENCE
}

Leandro G. Oliveira', Ana C. G. Lima1', Danilo A. Gusmão1, Deidimar C. B. Abreu', Frank L. B. Rodrigues', Alexandre Marchiori', Juarez A. Sousa', Rosemar M. S. Rahal' ${ }^{1}$

IInstituto Goiano de Oncologia e Hematologia - Goiânia (GO), Brazil.

Objectives: To describe the anatomopathological characteristics and the access to genetic testing among breast cancer young women attended at a private institution in Goiás-Brazil. Methodology: In this descriptive and retrospective study, were reviewed medical records of breast cancer young women ( $<$ or $=40$ years at the diagnosis) attended at a private cancer institution from March 2016 to March 2018. Data with respect to age at diagnosis, histopathological and immunohistochemical aspects and access to genetic testing were examined. Results: A total of 67 women were identified. The median age at the diagnosis was 36 years (range 26-40 years). The most common histological type was invasive ductal carcinoma (98.5\%); 45\% (n=30) and 41\% (n=28) were grade 3 and 2 tumours respectively. $70 \%(n=47)$ were estrogen receptor (ER) positive (at least $1 \%$ of positivity). $20,8 \%(n=14)$ were triple-negative and 2 women had ER positivity between 1-10\%. 26\% ( $\mathrm{n}=18)$ were human epidermal growth factor receptor -2 (HER-2) positive (HER-2 3+ or amplified FISH/ISH). The ki-67 index was $>14 \%$ in 51 women (76\%). Overall, 39\% ( $\mathrm{n}=26)$ got a genetic testing. The mean age of tested patients was 33,8 years. Among the women with available genetic results so far (n=19), six were identified (31\%) with pathogenic mutations (BRCA-1= 4; BRCA2+MUTYH=1; SKT11=1). Three of BRCA-1 mutated were triple-negative and the fourth had low hormonal receptor positivity (1\%). Conclusion: In this real-world and single-institution study, the proportion of triple negative and HER2 positive women was discretely higher than that described for older breast cancer patients. As expected, the majority of women had high grade tumours. A hereditary predisposition is more common amongst young women and may impact decisions on local and systemic disease management. Only the minority of this clinically high-risk group got a genetic testing. In this brief analysis, it was observed that almost one in four tested women harbored a pathogenic genetic mutation. Despite of the efforts to reduce the cancer care disparities, including the access to genetic counseling, significant barriers persist even among patients in private health assistance. Keywords: Breast cancer in young women; genetic testing; BRCA mutation. 